\title{
ABC of Computing
}

\section{AUXILIARY STORAGE}

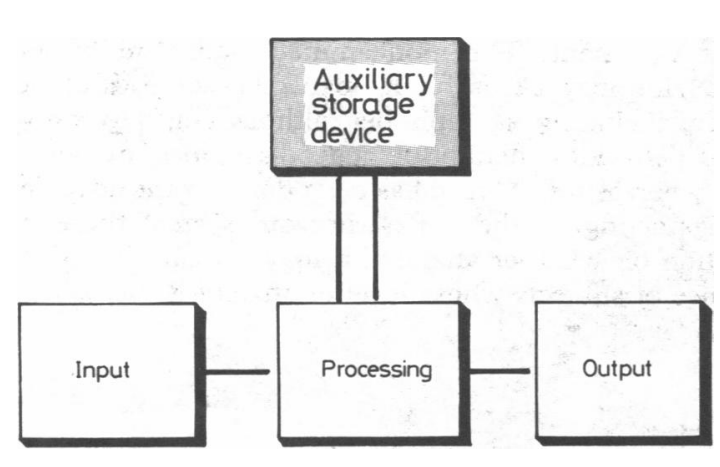

\section{Types of storage}
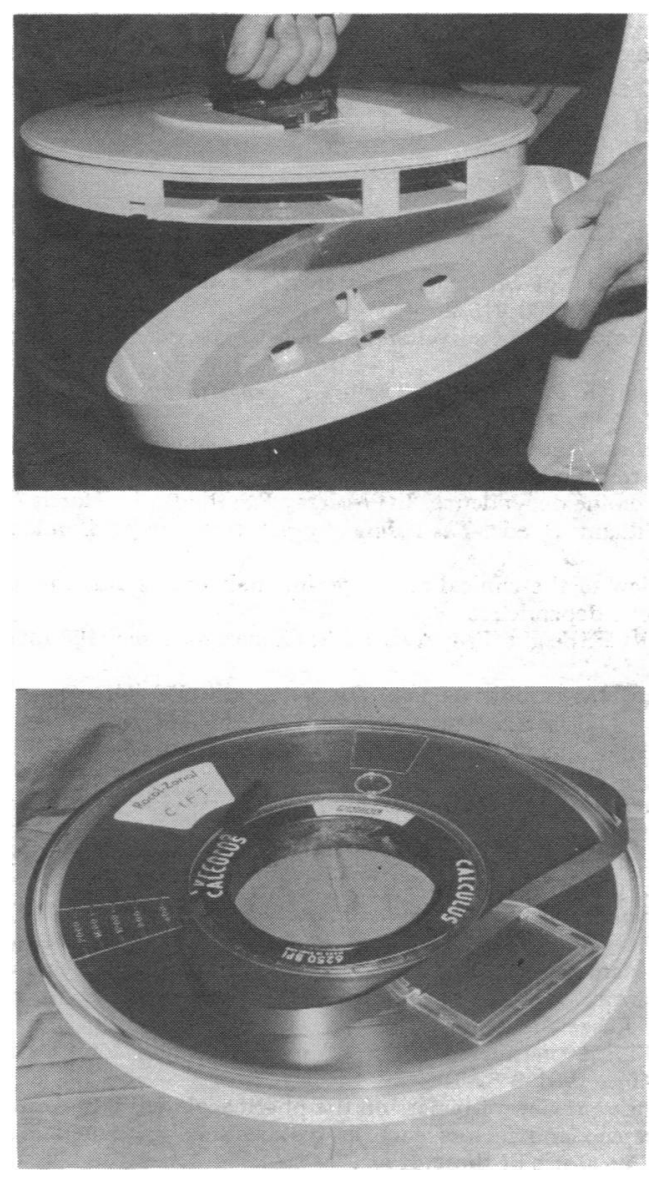

Auxiliary storage devices are the final part of the computer viewed as a four component system.

Time saving in computer work is economically important, and considerable savings can be made if the computer has all the required information available in computer readable form. The processing is not then held up by the user who is slow in entering data or by delays on a slow printer.

Auxiliary storage devices are under the control of the computer and hold information in a computer readable form in such a way that the computer can rapidly call the information into its own volatile memory. Information stored in the auxiliary device is retained when the computer is switched off and can be changed as often as necessary.

There are three main forms of auxiliary storage device: the magnetic tape, the hard disc, and the floppy disc.

Magnetic tape is one of the older storage media which has suddenly become popular again with the use of cassette tapes for microcomputers. The tape used in mainframe and minicomputer installations is wider, stronger, and of better quality than ordinary magnetic tape for domestic use and is usually mounted in a tape drive unit. This unit works under the control of the computer and can rapidly spool through 2400 feet of tape, bringing the required information to the tape read head within minutes. The movement of the tape has to be very precise because the computer readable characters-the $1 \mathrm{~s}$ and $0 \mathrm{~s}$ - are packed with a density of at least 1500 per inch of tape. If the wrong series of digits arrives at the tape read head the information in the whole tape may be misread. When such precise manipulation is needed the environmental humidity, static, and dust content must be controlled carefully or the tapes could be stretched or scratched by dust particles.

A $2400 \mathrm{ft}$ length of magnetic tape costing about $£ 10$ can store up to 30 megabytes of information, which is roughly equivalent to 3000 pages of text typed double spaced on A4 paper.

The cassette holds domestic quality magnetic tape and can do to a lesser degree for the microcomputer what the wider tapes do for mainframe machines. Information storage is much less reliable on domestic quality cassettes but they are cheap. They are commonly used by software manufacturers to distribute their programs in a computer readable form, and many micros have a cassette tape drive as a standard feature so that users can record their own programs.

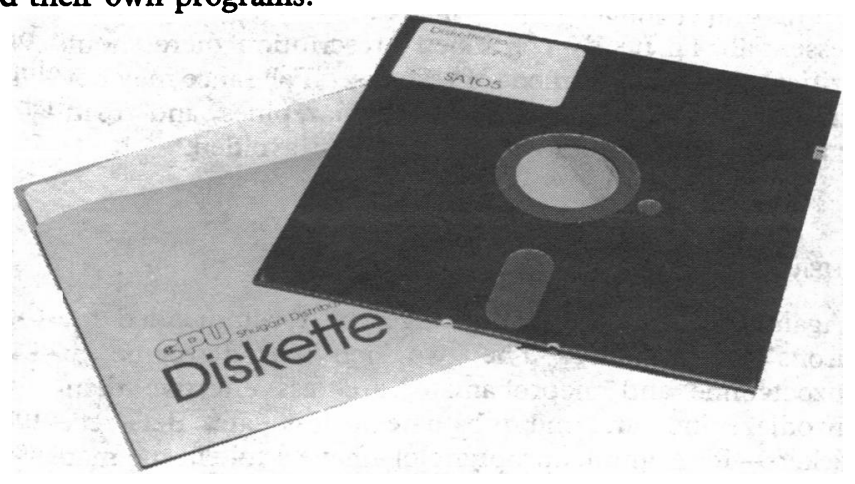



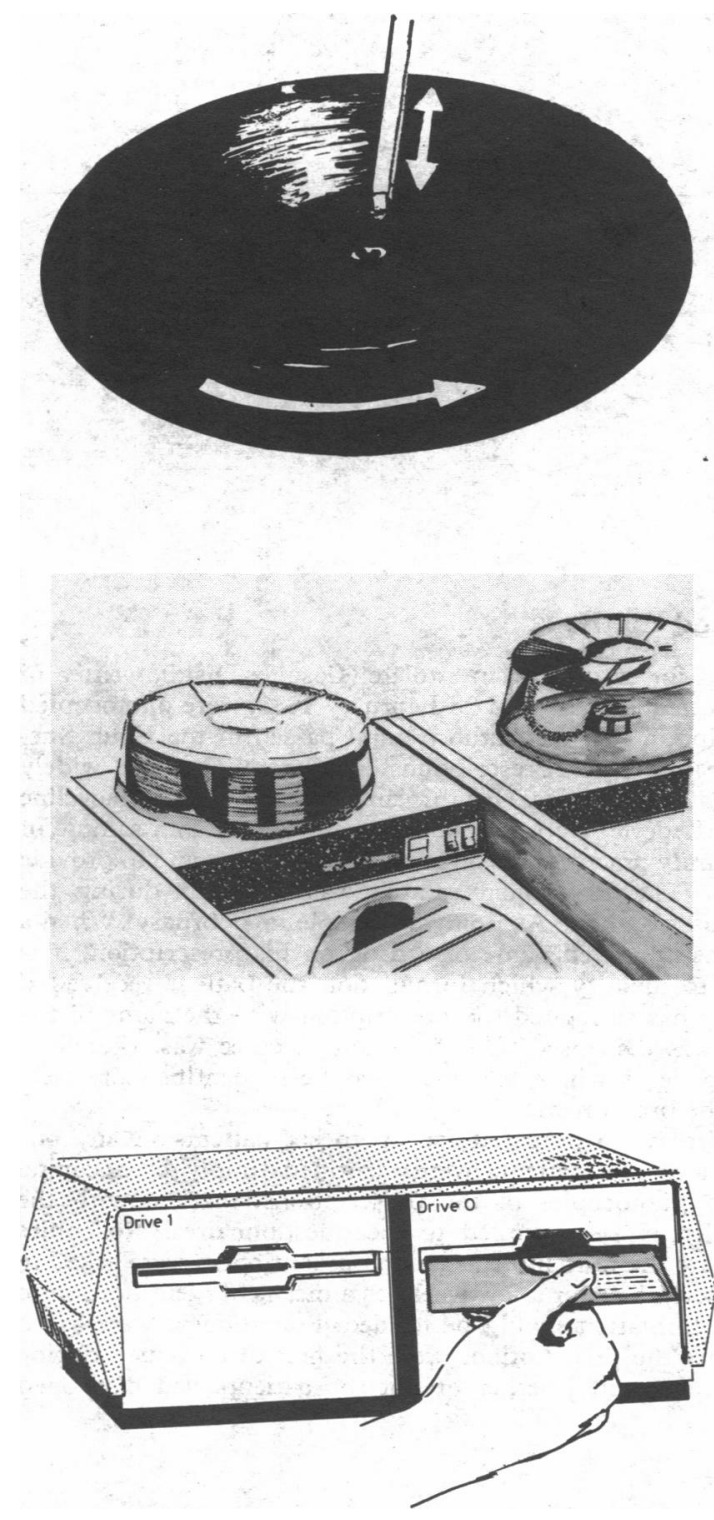

\section{Random and sequential access}

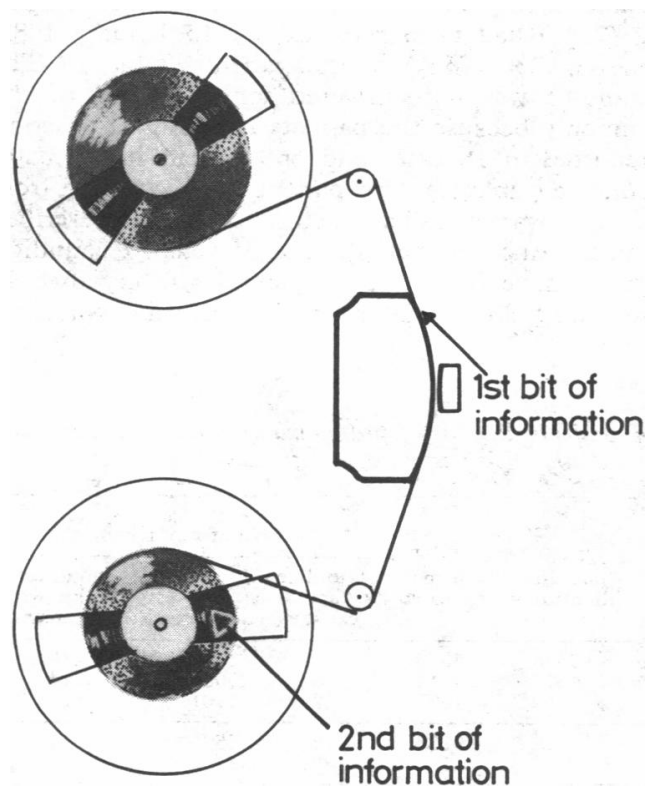

Disc storage involves a different concept from the magnetic tape. The hard disc is a 16-inch diameter circular sheet of inflexible ferromagnetic material mounted on a vertical spindle, and the information is recorded in segments on both surfaces. The disc revolves in an evacuated disc drive unit at speeds of over $2000 \mathrm{rpm}$, and the read head moves radially over the disc so that any piece of information is little more than one disc revolution away. A program need not wait more than a few milliseconds for its information, and processing can be done very rapidly.

Normally discs are mounted in groups of 10 comprising a disc assembly costing about $£ 500$ and having a combined capacity of over 300 megabytes. The disc drive unit with its ancillary equipment would normally cost over $£ 20$ 000. A large installation may have one disc drive unit, which could serve the needs of one minicomputer and 10 to 15 users. This could hold the stock records of a company, keeping track of the sales of several hundreds of products.

Floppy discs are flexible discs of ferromagnetic material usually 5 or 8 inches in diameter which can be used to store information in the same way as a hard disc. They are, however, cheaper than hard discs, costing about $£ 3$ each and having a storage capacity of 7-120 kilobytes. Although much stronger than the big discs and capable even of being sent through the post, they are as susceptible to strong magnetic fields as any magnetic medium. There have been several cases of information being erased from discs because they have been carried unprotected on underground trains. The disc drive used with floppy discs usually costs under $£ 1000$ and is often fitted as standard on many modern microcomputers.

A recent development is the availability of sealed fixed disc systems (Winchester discs) designed to work with microcomputers. The principle of use is the same as the hard disc, but the storage capacity is more limited, 10 megabytes being a common maximum. The whole unit comes sealed, so that the delicate disc surface is protected. A Winchester disc unit may cost about $£ 3000$.

The storage capacity of a sealed unit is undoubtedly useful, but as so much information can be stored, it is difficult to maintain a practical safety copy in case of failure. The information on the sealed disc may well fill many floppy discs and waste a lot of time in the process. One solution is to have duplicate sealed disc units.

Floppy discs are largely the province of the microcomputer user, but many large mainframe installations have facilities to read floppy discs. This facility is particularly useful as the user can collect and edit his data using his own microcomputer and then pass it to the mainframe for more powerful analysis-for example, by a statistical program that is not available on microcomputers.

One of the main problems with using tape to store data, such as a list of names, is that if the names are widely separated on the tape the tape has to be spooled backwards and forwards to find them, thereby wasting valuable seconds of computer time. This form of sequential access has obvious disadvantages and is suitable only when a tape can be read once and the information held temporarily in volatile memory within the processor itself, but this depends on the memory being large enough. A sorting operation to arrange patients' names in alphabetical order, though an ideal task for a computer, may take a long time if the data set on tape is large and the memory small.

Disc storage has now made random access economically viable, as the combination of revolving disc and the radially moving head makes all the data available within milliseconds, irrespective of their position. The difference is such that a receptionist at a hospital admission desk can type a patient's name into the computer, and within seconds (rather than minutes) the details of the patient already held on the disc can be displayed.

Dr A J Asbury, FFARCS, PHD, is lecturer in anaesthetics, University of Sheffield. 